\title{
The Pelagic Bacterium Paraphotobacterium marinum Has the Smallest Complete Genome Within the Family Vibrionaceae
}

\author{
Zhaobin Huang 1,2,3,4, Zhen Liu ${ }^{5}$ and Zongze Shao 1,2,3,4* \\ ${ }^{1}$ Key Laboratory of Marine Genetic Resources, Third Institute of Oceanography, State of Oceanic Administration, Xiamen, \\ China, ${ }^{2}$ Fujian Key Laboratory of Marine Genetic Resources, Xiamen, China, ${ }^{3}$ Fujian Collaborative Innovation Center of \\ Marine Biological Resources, Xiamen, China, ${ }^{4}$ South China Sea Bio-Resource Exploitation and Utilization Collaborative \\ Innovation Center, Xiamen, China, ${ }^{5}$ Shanghai Majorbio Bio-Pharm Biotechnology Co., Ltd., Shanghai, China
}

\section{OPEN ACCESS}

Edited by:

George S. Bullerjahn, Bowling Green State University,

United States

Reviewed by:

Kiilyukia Matthews Ciira, Mount Kenya University, Kenya Maria Cristina Thaller, Università degli Studi di Roma Tor Vergata, Italy

Christopher John Grim, United States Food and Drug Administration, United States

*Correspondence: Zongze Shao shaozz@163.com

Specialty section: This article was submitted to Aquatic Microbiology,

a section of the journal

Frontiers in Microbiology

Received: 21 July 2017 Accepted: 28 September 2017

Published: 11 October 2017

Citation:

Huang Z, Liu Z and Shao Z (2017)

The Pelagic Bacterium

Paraphotobacterium marinum Has the Smallest Complete Genome Within the Family Vibrionaceae.

Front. Microbiol. 8:1994. doi: 10.3389/fmicb.2017.01994
Members of the family Vibrionaceae are metabolically versatile and ubiquitous in natural environments, with extraordinary genome feature of two chromosomes. Here we reported the complete genome of Paraphotobacterium marinum NSCS20N07D ${ }^{\top}$, a recently described novel genus-level species in the family Vibrionaceae. It contained two circular chromosomes with a size of 2,593,992 bp with $\mathrm{G}+\mathrm{C}$ content of $31.2 \mathrm{~mol} \%$, and a plasmid with a size of 5,539 bp. The larger chromosome (Chr. I) had a genome size of 1,426,504 bp with $\mathrm{G}+\mathrm{C}$ content of $31.6 \mathrm{~mol} \%$, and the smaller one (Chr. II) had a genome size of 1,161,949 bp with $\mathrm{G}+\mathrm{C}$ content of $30.8 \mathrm{~mol} \%$. The two chromosomes have strikingly similar $\mathrm{G}+\mathrm{C}$ contents with difference of $<1 \%$ and similar percentages of coding regions. Interestingly, by comparison to 134 species affiliated with seven genera within the family Vibrionaceae, P. marinum NSCS20N07D ${ }^{\top}$ possessed the smallest genome size and lowest $\mathrm{G}+\mathrm{C}$ content. Clusters of orthologous groups of proteins functional categories revealed that the two chromosomes had different distributions of functional classes, indicating they take different cellular functions. Surprisingly, Chr. II had a large proportion of unknown genes than Chr. I. Metabolic characteristics predicted that Chr. I performed the essential metabolism, which can be complemented by the Chr. II, such as amino acids biosynthesis. Microbial community analysis of in situ surface seawater revealed that $P$. marinum accounted for one to four sequences among more than 20,000 of $16 \mathrm{~S}$ ribosomal RNA gene V4 contigs, representing it apparently appeared as a rare species. What's more, $P$. marinum was anticipated to be specific to the pelagic ocean. This study will provide new insight into more understanding the genomic and metabolic features of multiple chromosomes in prokaryote and emphasize the ecological distribution of the members in the family Vibrionaceae as a rare species.

Keywords: Paraphotobacterium marinum, complete genome, two chromosomes, metabolic features, rare species

\section{INTRODUCTION}

Members of the family Vibrionaceae are metabolically versatile, and widely distributed in marine environments, either free-living or associated with marine animals. Interestingly, it is reported that members of the family Vibrionaceae have an extraordinarily ubiquitous genome feature that they possess two chromosomes (Okada et al., 2005; Dryselius et al., 2007; Dikow and Smith, 2013). 
The cholera pathogen Vibrio cholerae El Tor N16961, for example, contains two chromosomes, with the large one of 3.0 Mbp and the small one of $1.1 \mathrm{Mbp}$ (Heidelberg et al., 2000). Because $V$. cholerae is the most clinically important vibrio causing cholera, a severe diarrheal disease, it becomes a model organism to study the chromosome replication initiation (Baek and Chattoraj, 2014; Gerding et al., 2015), cell division mechanisms (Galli et al., 2016), and other fundamental biological investigations. Vibrio parahaemolyticus KX-V237, another pathogenic vibrio causing gastroenteritis, also contains two circular chromosomes, with the large one of $3.3 \mathrm{Mbp}$ and the small one of 1.9 Mbp (Tagomori et al., 2002). Although the sizes of the large chromosomes compared between the species $V$. cholerae and $V$. parahaemolyticus are similar, the sizes of the small ones are different (Tagomori et al., 2002). The considerably variable size of the small chromosome of the vibrios (0.8-2.4 Mbp) becomes a common genome feature of members of the family Vibrionaceae (Okada et al., 2005; Dryselius et al., 2007).

PacBio RS sequencing platform is called as the thirdgeneration sequencer, which is a single-molecule, real-time sequencing technology that does not need to amplify the genomic DNA, and thus it can produce sequences with longer read length (Eid et al., 2009). By application of the PacBio RS sequencing platform, the complete and closed genome of Burkholderia pseudomallei with two large and complex circular chromosomes and high $\mathrm{G}+\mathrm{C}$ content (68-69\%) is obtained fastly and accurately (Teng et al., 2017).

We identify and describe a new genus-level species within the family Vibrionaceae, named Paraphotobacterium marinum, which is isolated from the surface seawater of the South China Sea (Huang et al., 2016). Interestingly, the genome size of P. marinum NSCS20N07D ${ }^{\mathrm{T}}$ is $2.5 \mathrm{Mbp}$ calculated from the draft genome sequence, which is much more smaller than the closely related members within the family Vibrionaceae (Huang et al., 2016). Here we reported the whole complete genome of $P$. marinum NSCS20N07D ${ }^{\mathrm{T}}$ using PacBio RS sequencing platform, and the metabolic features were predicted with genome annotation. In addition, we performed the microbial diversity analysis of the in situ surface seawater where $P$. marinum NSCS20N07D ${ }^{\mathrm{T}}$ was isolated, aiming to investigate the relative abundance of $P$. marinum in natural environment. Finally, we predicted the natural distribution of $P$. marinum. This study provides new insights into more understanding the genomic and metabolic features of the Vibrionaceae member, Paraphotobacterium containing two chromosomes and the ecological distribution in the ocean.

\section{MATERIALS AND METHODS}

\section{Genomic DNA Extraction and Quality Assessment}

Paraphotobacterium marinum NSCS20N07D ${ }^{\mathrm{T}} \quad(=\mathrm{KCTC}$ $52126^{\mathrm{T}}=$ MCCC $\left.1 \mathrm{~A} 01886^{\mathrm{T}}=\mathrm{CIP} 111031^{\mathrm{T}}\right)$ was cultured with Marine Broth 2216 (BD, Difco). The cell biomass of $100 \mathrm{ml}$ was obtained by centrifugation at $6,000 \times g$ for $10 \mathrm{~min}$.
The genomic DNA was extracted using the conventional phenol/chloroform/isoamyl alcohol (25:24:1) extraction method. The completeness of the extracted DNA was tested using $1.0 \%$ agarose gel electrophoresis. The concentration and purity were determined using TBS-380 and NanoDrop 2000 spectrophotometer.

\section{PacBio Sequencing and Sequence Assembly}

A $10 \mathrm{~kb}$ DNA fragment library was constructed according to the manufacturer's instruction and sequenced on the PacBio RSII sequencing platform with one SMART cell (MajorBio Co., Shanghai, China). Sequencing reads were assembled using the hierarchical genome-assembly process (HGAP 3.0) (Chin et al., 2013). A complete genome with two chromosomes and one plasmid of $P$. marinum NSCS20N07D ${ }^{\mathrm{T}}$ was obtained.

\section{Genome Annotation}

The prediction of the protein coding genes (opening reading frames, ORFs) was carried out using Glimmer 3.0 (Delcher et al., 2007). The ribosomal RNA (rRNA) genes were predicted using Barrnap $^{1}$. The tRNA genes were predicted using tRNAscan-SE (Lowe and Eddy, 1997). Gene annotation was carried out by BLASTP search against non-redundant protein sequences (nr database), Swissprot database, the clusters of orthologous groups of proteins (COG) database, and KEGG database (Kanehisa et al., 2016) with $e$-value cutoff of $1 \mathrm{e}-5$ (Tatusov et al., 2001). Further annotation was performed using the RAST server online (Aziz et al., 2008) and MicroScope platform (Vallenet et al., 2009). The circular genome was drawn using Circos (Krzywinski et al., 2009).

\section{Microbial Composition of the in Situ Surface Seawater and Relative Abundance of $\boldsymbol{P}$. marinum}

The in situ surface seawater of two sites (NSCS1 [119 $26^{\prime} 24.62^{\prime \prime} \mathrm{E}$, $\left.21^{\circ} 58^{\prime} 35.71^{\prime \prime} \mathrm{N}\right]$ and NSCS2 [117 $\left.\left.36^{\prime} 11.76^{\prime \prime} \mathrm{E}, 19^{\circ} 19^{\prime} 17.30^{\prime \prime} \mathrm{N}\right]\right)$ in the South China Sea was collected on May 14-15, 2015. P. marinum NSCS20N07D ${ }^{\mathrm{T}}$ was isolated from site NSCS2 at a depth of 3,048 $\mathrm{m}$ (Huang et al., 2016). Triplicate 21 seawater of each sampling site was filtered on board using $0.22 \mu \mathrm{m}$ filter membrane (GF, Millipore, United States). The membrane was put into a $2.0 \mathrm{ml} \mathrm{EP}$ tube and store at $-20^{\circ} \mathrm{C}$ until use.

The total DNA of filtered surface seawater was extracted using the traditional proteinase K/SDS lysis followed by phenol/chloroform/isoamyl alcohol (25:24:1) extraction method (Huang et al., 2014). The quality of total DNA was evaluated by $1.0 \%$ agarose gel electrophoresis. The $16 \mathrm{~S}$ rRNA gene V4 region was amplified with the specific primers 515F (GTGCCAGCMGCCGCGGTAA) and 806R (GGACTACHVGGGTWTCTAAT) with dual barcode (Kozich et al., 2013). The PCR amplification mixture was made in $50 \mu \mathrm{l}$ reactions with total DNA of $10 \mathrm{ng}$. The PCR reaction was conducted with TransStart Fastpfu DNA Polymerase as follows: initial denaturation at $98^{\circ} \mathrm{C}$ for $1 \mathrm{~min}$, followed by 30 cycles of

${ }^{1}$ http://www.vicbioinformatics.com/software.barrnap.shtml 
denaturation at $98^{\circ} \mathrm{C}$ for $10 \mathrm{~s}$, annealing at $55^{\circ} \mathrm{C}$ for $30 \mathrm{~s}$ and elongation at $72^{\circ} \mathrm{C}$ for $30 \mathrm{~s}$, and extension at $72^{\circ} \mathrm{C}$ for $5 \mathrm{~min}$. For each sample, three parallel PCR reactions were carried out and pooled together before purification of the PCR product with AxyPrepDNA gel extraction Kit (Axygen). The high-throughput sequencing of the $16 \mathrm{~S}$ rRNA gene V4 region was performed using Illumina Miseq PE250 platform (Illumina, San Diego, CA, United States).

The pair-end reads of each sample were merged into contigs using make.contigs command implemented in mothur v.1.34.4 (Schloss et al., 2009). The contigs were then trimmed using Qiime pipeline (Caporaso et al., 2010) as follows: (1) allowing no mismatch with dual barcode and primers; (2) allowing no ambiguous bases; and (3) removal of contigs containing the homopolymers over 6 . Then, the chimeras were checked using usearch61 algorithm against the latest Greengenes database of gg_13_8_97_otus (DeSantis et al., 2006). The operation taxonomic unit (OTU) was picked with a 97\% similarity threshold using the de novo picking method.

The contigs of V4 region of each sample affiliated with $P$. marinum were identified by mapping to the full-length $16 \mathrm{~S}$ rRNA gene of $P$. marinum NSCS20N07D ${ }^{\mathrm{T}}(1,539 \mathrm{bp}$, accession number: KU140414) using BLASTN with $e$-value of $1 e-5$ and sequence similarity of $>97 \%$.

\section{Ecological Distribution of $\boldsymbol{P}$. marinum}

The closely related sequences of $P$. marinum were searched in GenBank using Nucleotide BLAST using the full-length $16 \mathrm{~S}$ rRNA gene of $P$. marinum NSCS20N07D ${ }^{\mathrm{T}}$. The sequence similarity above $97 \%$ with $P$. marinum NSCS20N07D ${ }^{\mathrm{T}}$ was determined as the same species, and the isolation source of the closely related sequences was summarized.

\section{RESULTS AND DISCUSSION}

\section{Genome Features of $P$. marinum NSCS20N07D ${ }^{\top}$}

A total of 131,203 clean reads were obtained with the largest of $40,999 \mathrm{bp}$ retrieved from one SMART cell of PacBio RSII sequencing. The complete genome of $P$. marinum NSCS20N07D ${ }^{\mathrm{T}}$ was obtained, containing two circular chromosomes with a total size of $2,593,992$ bp with $\mathrm{G}+\mathrm{C}$ content of $31.2 \mathrm{~mol} \%$, and one plasmid with a size of 5,539 bp. The larger chromosome (Chr. I) had a genome size of 1,426,504 bp with $\mathrm{G}+\mathrm{C}$ content of $31.6 \mathrm{~mol} \%$, and the smaller one (Chr. II) had a genome size of $1,161,949$ bp with $\mathrm{G}+\mathrm{C}$ content of $30.8 \mathrm{~mol} \%$ (Figure 1 and Table 1).

By comparison to 134 species affiliated with seven genera in the family Vibrionaceae, $P$. marinum NSCS20N07D ${ }^{\mathrm{T}}$ possessed the smallest genome size and lowest G+C content (Figure 2). As far as we know, $P$. marinum NSCS20N07D ${ }^{\mathrm{T}}$ was the smallest genome in Vibrionaceae species reported so far.

To compare $P$. marinum with other Vibrionaceae species in more details on each chromosomes, we selected seven representative species with complete genome, Photobacterium profundum SS9, Photobacterium gaetbulicola Gung47, Alivibrio fischeri ES114, Aliivibrio salmonicida LFI1238, V. cholerae O1 biovar El Tor str. N16961, V. parahaemolyticus RIMD 2210633, and Grimontia hollisae ATCC 33564 (Table 1). Obviously, Chr. I of $P$. marinum NSCS20N07D ${ }^{\mathrm{T}}$ was the smallest chromosome among those of the above representative species, the size of which ranged from 2,897,536 bp of A. fischeri ES114 to 4,085,304 bp of P. profundum SS9. Strangely, Chr. II of P. marinum NSCS20N07D ${ }^{\mathrm{T}}$ was not the smallest. The size of the smaller chromosome showed considerably variable pattern among the compared members of the family Vibrionaceae, ranging from 783,080 to 2,237,943 bp (Table 1). The variable size of Chr. II was recognized as more flexible genes therein (Okada et al., 2005). G+C content of the two circular chromosomes of P. marinum NSCS20N07D ${ }^{\mathrm{T}}$ had less than $1 \%$ difference. This was in congruence with the genome characteristics of Vibrionaceae species, which was regarded as a long evolutionary co-existence for the divided chromosomes (Okada et al., 2005). Furthermore, on the aspect of genome size, the difference between Chr. I and Chr. II was much smaller in P. marinum NSCS20N07D ${ }^{\mathrm{T}}$ than the corresponding other species within this family (Table 1).

Through gene annotation, we found that Chr. I encoded 1,481 ORFs, and had 8 copies of $r r n$ operon (16S-23S-5S rRNA genes), an extra 5S rRNA gene, and 69 tRNA genes; while Chr. II encoded 1,148 ORFs and had 1 copy of $r r n$ operon and 7 tRNAs. It was noteworthy that all these $16 \mathrm{~S}$ rRNA genes shared 100\% sequence identity (Figure 1 and Table $\mathbf{1}$ ).

By means of BLASTP search against $\mathrm{nr}$ database, Chr. II had a larger proportion of unknown proteins and hypothetical proteins, accounting for 29.4 and $29.8 \%$, respectively, indicating there was a larger proportion of genes not yet elucidated (Supplementary Table S1).

\section{COG Categories}

By COG functional annotating, we found that $42.47 \%$ in $\mathrm{Chr}$. I and $13.50 \%$ in Chr. II can be annotated. Surprisingly, a huge amount of ORFs (86.50\%) in Chr. II could not be classified (No assigned COG in Figure 1). This was in congruence with the result of BLASTP against $n r$ database, indicating that the biological function of most genes in Chr. II was still unknown. On the other hand, the vast majority of genes for essential cell function were located in Chr. I determined from COG classes, such as Class J of translation, ribosomal structure, and biogenesis; Class $\mathrm{C}$ of energy production and conversion; Classes of D, E, F, G, and P of transport; and Class L of replication, recombination, and repair. Cell motility and secretion (Class N) were undertaken mainly by Chr. I other than Chr. II. COG functional categories revealed that the two chromosomes have different distributions of functional classes, indicating they take different cellular functions.

\section{Replication Initiation}

The study on chromosome replication of the two chromosomes of $V$. cholerae attracted much more attention in recent years (Baek and Chattoraj, 2014; Val et al., 2016). DnaA was reported as the main controller in Chr. I in $V$. cholerae (Baek and Chattoraj, 2014). Similarly, in P. marinum NSCS20N07D ${ }^{\mathrm{T}}$, the replication initiation region of Chr. I was identified 


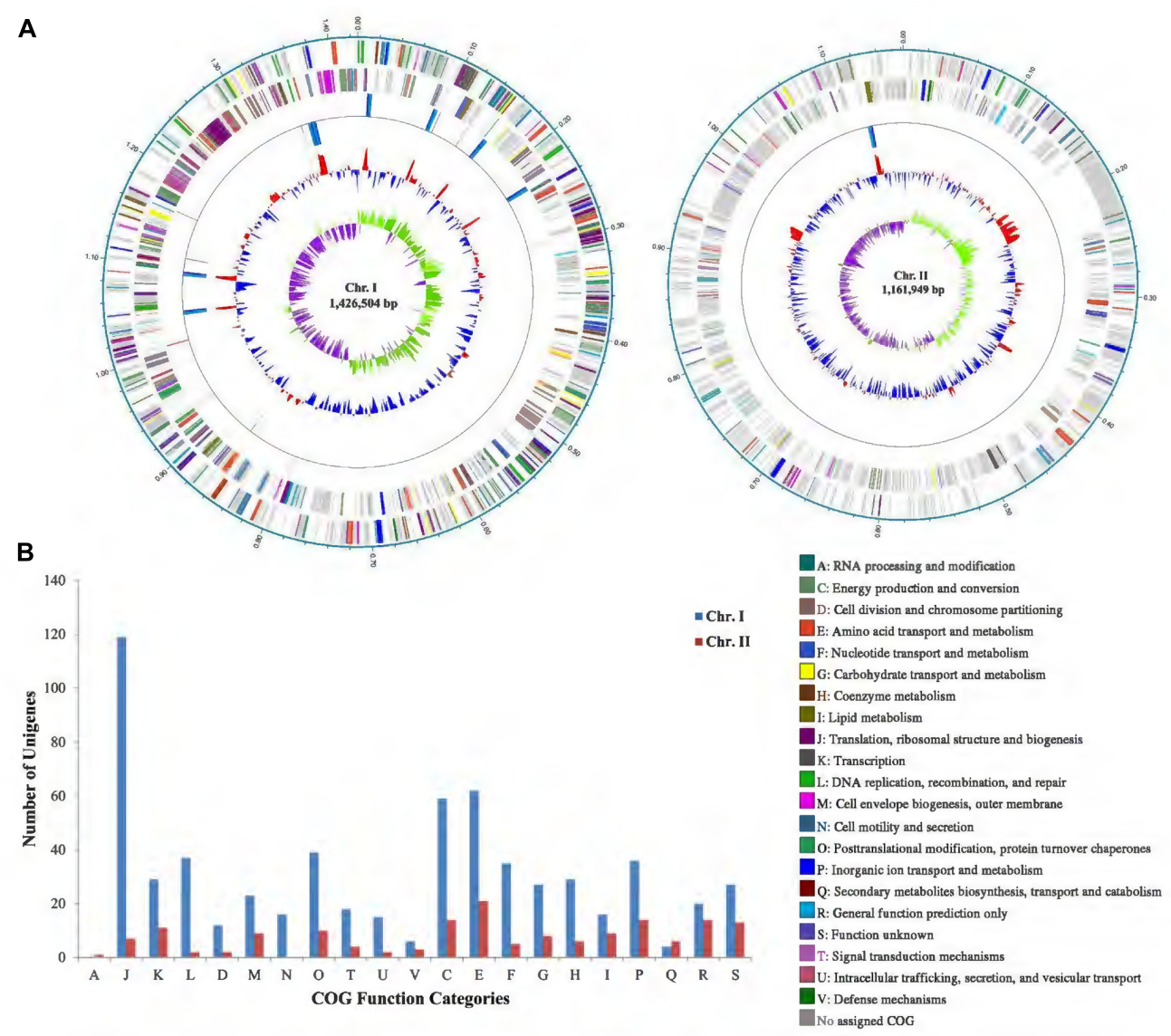

FIGURE 1 | (A) Circular genome maps of P. marinum NSCS20N07D ${ }^{\top}$ containing two chromosomes. From the inside to outside: GC-skew; GC content; $r$ rn operon; and tRNA genes; ORFs on the reverse strand (colorized by COG functional categories); ORFs on the forward strand (colorized by COG functional categories); locations in megabases (Mb). (B) COG functional categories of annotated ORFs in Chr. I and Chr. II of $P$. marinum NSCS20N07D'. COG included three large groups: information storage and processing (A, J, K, L); cellular processes and signaling (D, M, N, O, T, U, V), metabolism (C, E, F, G, H, I, P, Q), and poorly characterized (R, S).

by the gene configuration of $d n a A, d n a N$, and $r e c F$, which encoded chromosomal replication initiation protein DnaA, DNA polymerase III subunit beta, and DNA replication/repair protein RecF, respectively (Supplementary Table S1). CrtS, the RctB binding locus, was reported to mediate the replication initiation of Chr. II (Baek and Chattoraj, 2014). However, in P. marinum, we did not find $\mathrm{CrtS}$, whereas the replication initiation region of Chr. II was identified by chromosome partitioning protein ParB (orf00002_1) and protein ParA (orf00003_1). With respect to the plasmid replication, there was a RepA protein found in the plasmid of $P$. marinum, which can control the replication of plasmid.

\section{Carbohydrate Metabolism}

Complete genes taking part in the central carbohydrate metabolism could be found in Chr. I (Figure 3), including the Embden-Meyerhof-Parnas pathway (EMP or glycolysis), pyruvate oxidation to acetyl-CoA, tricarboxylic acid (TCA) cycle, and glyoxylate bypass (also called glyoxylate cycle). Chr. I also contained an Entner-Doudoroff pathway, which could replace the glycolysis to catabolize glucose to pyruvate. Most genes involved in pentose phosphate pathway were encoded in Chr. I, however, the transaldolase catalyzing the step of sedoheptulose 7-phosphate and glyceraldehyde 3-phosphate to form fructose 6phosphate and erythrose 4-phosphate was encoded in Chr. II. This indicated the pentose metabolism could be regulated by the smaller chromosome Chr. II.

On the aspect of utilization of carbon source, the two chromosomes hosted different genes encoding different metabolic activities. Chr. I possessed the genes encoding the utilization of D-glucose, while Chr. II mainly possessed those for the utilization of D-galactose. This demonstrated that Chr. II improved the ability of the metabolic range of carbon source. No data supported that this bacterium can utilize oligosaccharides.

\section{Amino Acids Biosynthesis and tRNAs}

Through metabolic pathway analysis, 13 kinds of amino acids can be synthesized, which were found in Chr. I. The majority of genes involved in arginine biosynthesis could be found in Chr. II except the gene $\arg D$ encoding acetylornithine aminotransferase, 


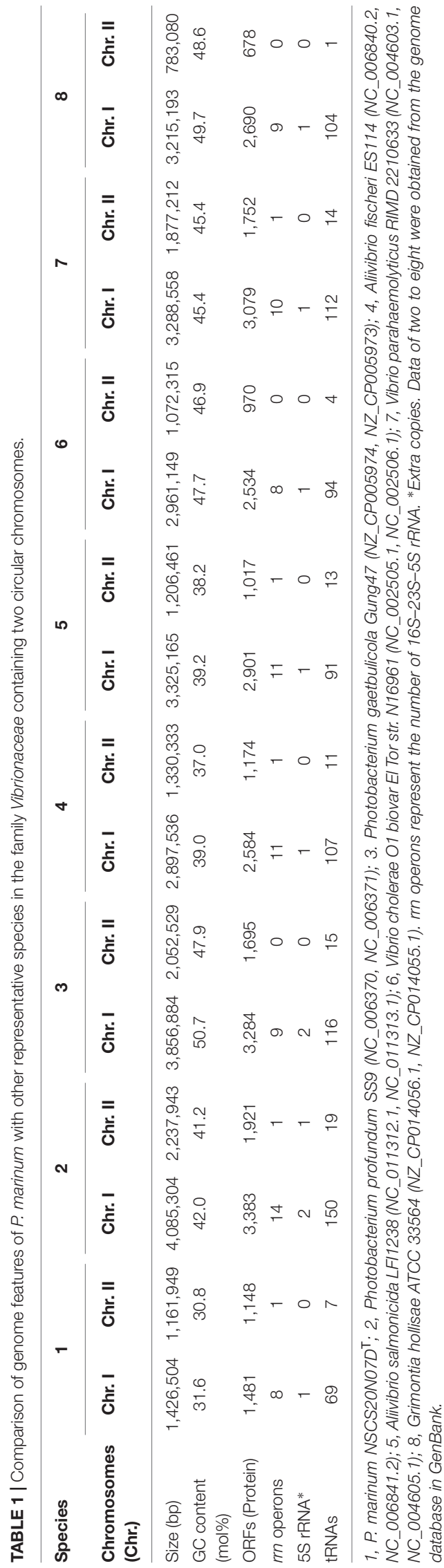

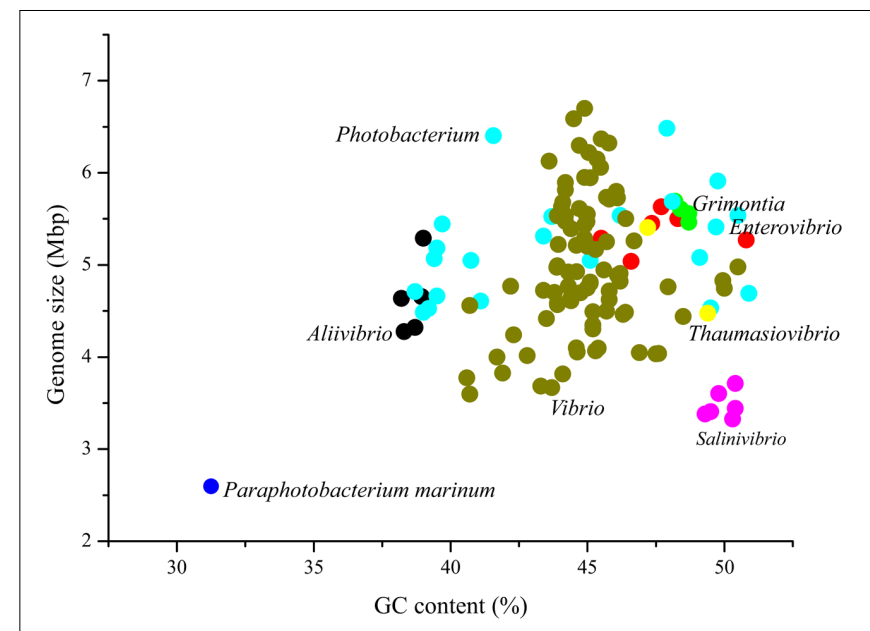

FIGURE 2 | Genome size and GC content of $P$. marinum NSCS20N07D ${ }^{\top}$ in comparison with other species in the family Vibrionaceae. There were 134 genomes available in the genome resource in NCBI database, belonging to the following seven genera, Aliivibrio (5), Enterovibrio (6), Grimontia (5), Photobacterium (22), Salinivibrio (6), Thaumasiovibrio (2), and Vibrio (88). The number in bracket indicated the number of genomes within each genus. Although there were two genomes smaller than $P$. marinum NSCS20N07D ${ }^{\top}$, "Candidatus Photodesmus blepharushas" of 1.11231 Mbp with GC content of $35.6 \%$, and "Candidatus Photodesmus katoptron" of $1.01592 \mathrm{Mbp}$ with GC content of $30.9 \%$, they were not described as a species, since they were organism-specific and uncultured yet. Thus, the two genomes were excluded in this study.

which was encoded in Chr. I (Supplementary Table S1). Tryptophan and proline biosynthesis were also annotated in the two chromosomes (Figure 3).

Through gene annotation, Chr. I contained 19 kinds of tRNA synthetase responsible for the 19 amino acids except proline. Chr. II contained only two tRNA synthetases for proline and tryptophan, respectively.

\section{Poly- $\beta$-Hydroxybutyrate Biosynthesis and Degrading Pathway}

Strain NSCS20N07D ${ }^{\mathrm{T}}$ can produce a large amount of poly$\beta$-hydroxybutyrate (PHB) granules inside the cell observed using transmission electron microscopy (TEM) after negative staining and Nile blue A stain (Huang et al., 2016). Gene annotation and metabolic analysis revealed that a gene cluster (atoB, $p h b B$, and $p h b C$ ) was present in Chr. II for PHB biosynthesis from two acetyl-CoA molecules to form acetoacetyl-CoA (Figure 3), whereas it seems that the degrading of PHB was controlled by Chr. I as the incomplete pathway from $(R)$-3-hydroxybutanoate to acetone was found, but the mechanism of polymer hydrolyze needed further study.

\section{Flagella}

All genes encoding for flagellar biosynthesis proteins could be found in Chr. I (Supplementary Table S1), indicating that strain NSCS20N07D ${ }^{T}$ could be motile. However, no flagella were observed by TEM after negative stain (Huang et al., 2016), making it likely that the flagella were lost in sample preparation. 


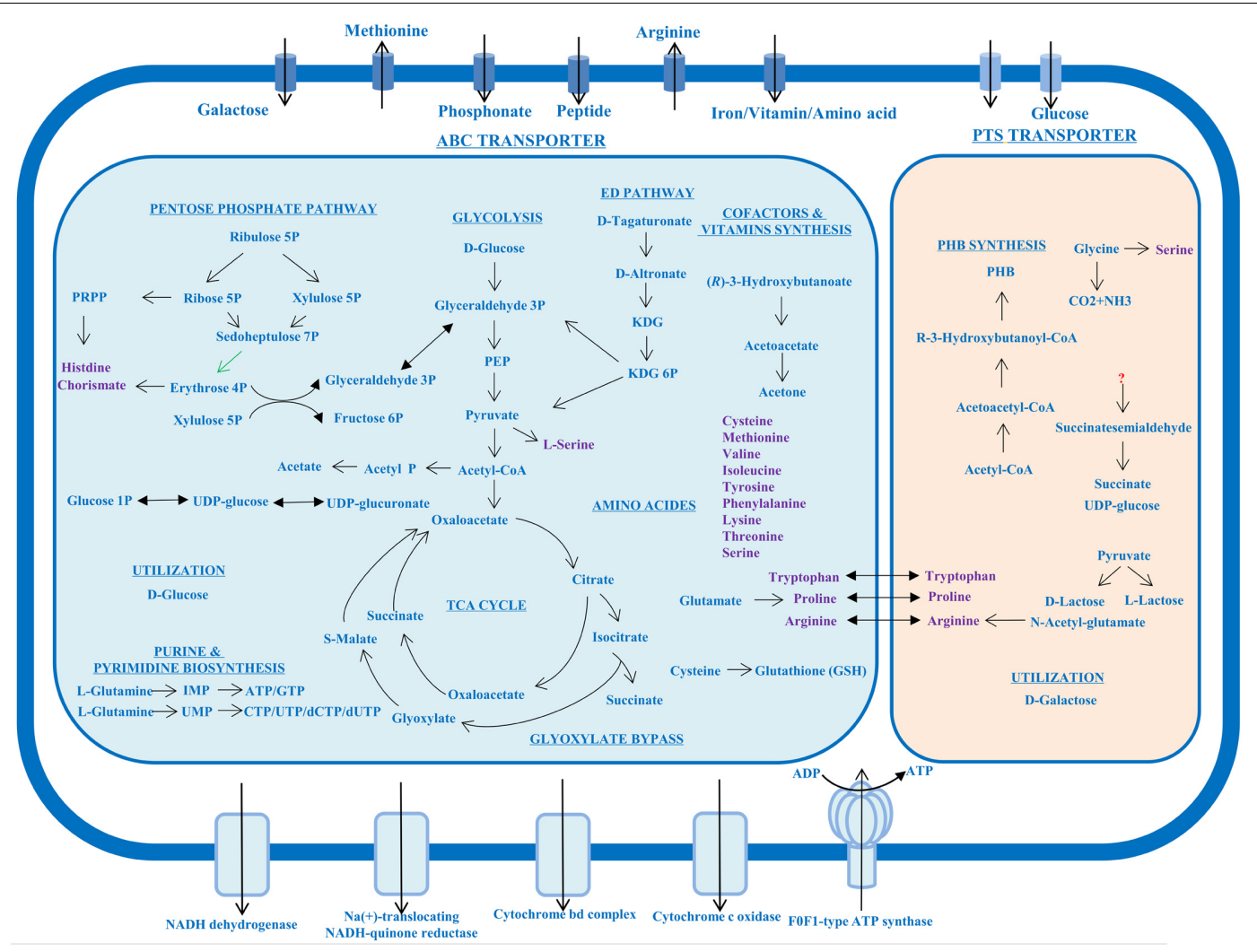

FIGURE 3 | Overview of metabolic characteristics of $P$. marinum NSCS20NO7D ${ }^{\top}$. The large blue box and the small orange box showed the metabolic pathway in Chr. I and Chr. II, respectively. Amino acids were marked in purple. The double sided arrows across the two boxes represented these amino acids synthesis genes were encoded in the two chromosomes. The green arrow represented the gene involved in this step was encoded in Chr. II. KDG, 2-dehydro-3-deoxy-D-gluconate; PEP, phosphoenolpyruvate; PHB, poly- $\beta$-hydroxybutyrate.

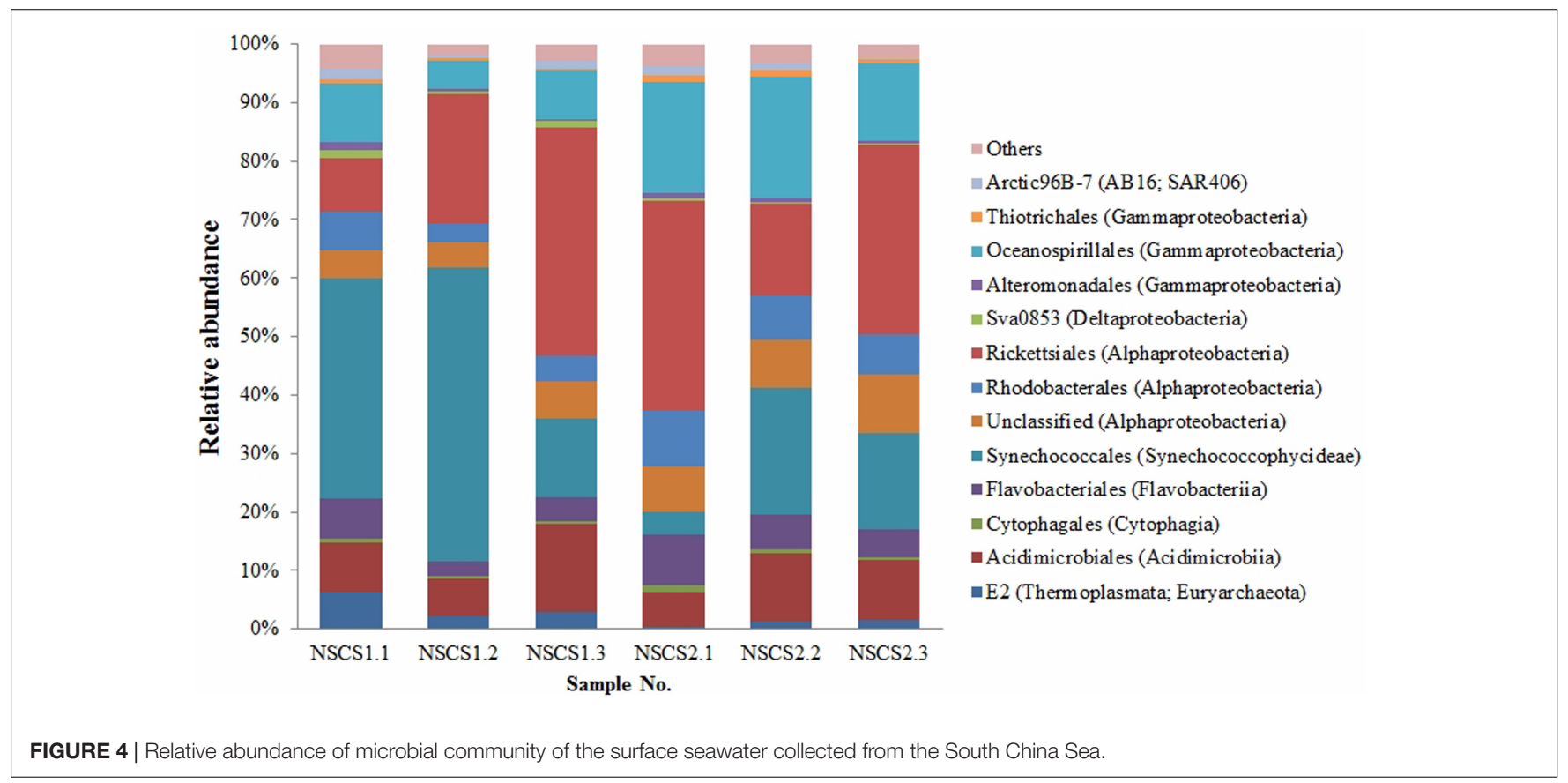




\section{Bacterial Relative Abundance of $P$. marinum in in Situ Surface Seawater}

To investigate the relative abundance of $P$. marinum in the surface seawater where it was isolated, we carried out microbial diversity analysis based on high-throughput sequencing of 16S rRNA gene V4 region using Miseq platform. The six samples produced a total of 142,564 contigs with the minimum of 18,669 contigs for NSCS1.3 and the maximum of 28,067 contigs for NSCS1.1. The surface seawater of the sampling sites in South China Sea was dominated by Cyanobacteria, Proteobacteria, Bacteroidetes, and Actinobacteria, though the data seemed to be variable among each samples (Figure 4). Synechococcales in Cyanobacteria (3.82-50.19\%), Rickettsiales in Alphaproteobacteria (9.10-38.90\%), Oceanospirillales in Gammaproteobacteria (4.97-20.89\%), and Acidimicrobiales in Actinobacteria (6.11-15.05\%) were the dominant groups in the surface seawater (Figure 4). Flavobacteriales of the class Flavobacteriia accounted for $2.47-8.74 \%$ relative abundance (Figure 4). While sequences affiliated with the family Vibrionaceae accounted for less than $0.1 \%$ of relative abundance.

By mapping the V4 contigs to the full-length 16S rRNA gene of $P$. marinum NSCS20N07D ${ }^{\mathrm{T}}$, we found that there were four contigs (253 bp in length) in NSCS1.1 (28,067 contigs), one contig in each sample of NSCS1.2 (25,062 contigs), NSCS2.1 (24,463 contigs), and NSCS2.2 (22,064 contigs), having 99.6$100 \%$ similarity with the $16 \mathrm{~S}$ rRNA gene of $P$. marinum, while other 16S rRNA gene V4 contigs showed the similarity no more than $96.0 \%$. The seven sequences were classified into a single OTU (denovo_289). The low relative abundance of $P$. marinum indicated that it was a rare bacterial species which were regarded as the threshold of five sequences per OTU (Skopina et al., 2016). Thus, it is assumed that P. marinum is present as a rare species in the surface seawater.

\section{Ecological Distribution of $P$. marinum}

Through 16S rRNA gene similarity search against the Nucleotide collection (nr/nt) database, we found that this bacterium P. marinum showed $99 \%$ similarity with the 21 uncultured bacterium clones detected from the Red Sea coral Pocillopora damicornis (accession number: KC668602), seawater from South China Sea (GU061990, GU061981), hydrothermal plumes of East Pacific Rise (JN874365, JN874376, JN874374, JN873939, JN874354, JN874093, JN874308, JN874318, JN874322), West Pacific (KF271041), and Gulf of Mexico oil sheen (KF786540, KF786512, KF786461), ocean water of north eastern Gulf of Mexico ( KU578418), and CALCOFI Line 67-155 depth of $5 \mathrm{~m}$ size fraction 3.0-20 $\mu \mathrm{m}$ (KX935264, KX935265, KX935266, KX935267). These environments, to some extent, are all pelagic niches that are distinct from the coast region. Thus, we speculate that $P$. marinum is specific to the pelagic environment, and has a global distribution by free-living, or coral-associated, and from surface to the deep extreme hydrothermal environments.

\section{CONCLUSION}

In conclusion, this is the first report of the complete genome sequence and metabolic features of $P$. marinum containing two chromosomes. By comparison to other members in the family Vibrionaceae on the aspect of genome features, P. marinum has the smallest genome reported so far. Metabolic characteristics predict that Chr. I and Chr. II have different genes, indicating they perform different cellular functions. A large proportion of unknown genes is present in the small chromosome, highlighting the main functions of Chr. II remain unclear. Finally, P. marinum is assumed to be specific to pelagic environment, and widely distributed in the global ocean as a rare species. Our study will provide new insights into further understanding the genomic features of Vibrionaceae members containing two chromosomes, and the multipartite metabolic features of multiple chromosomes in prokaryote, and emphasize the ecological distribution of Vibrionaceae species as a rare species.

\section{NUCLEOTIDE SEQUENCE ACCESSION NUMBERS}

The whole complete genome sequence of $P$. marinum NSCS20N07D ${ }^{\mathrm{T}}$ has been deposited in GenBank under accession number CP022355-CP022357.

\section{AUTHOR CONTRIBUTIONS}

$\mathrm{ZH}$ and $\mathrm{ZS}$ conceived this study. ZH performed the data analysis. ZL conducted sequence assembly and genome annotation. ZH and ZS wrote the manuscript.

\section{FUNDING}

This work was financially supported by the project of National Infrastructure of Microbial Resources of China (NIMR 2017-9), High-Tech Research and Development Program of China (No. 2012AA092102), and Innovation and Development of Marine Economy Demonstration Project of Xiamen City (16PZP001SF16).

\section{SUPPLEMENTARY MATERIAL}

The Supplementary Material for this article can be found online at: https://www.frontiersin.org/articles/10.3389/fmicb. 2017.01994/full\#supplementary-material

TABLE S1 | Genome annotation of the two chromosomes of $P$. marinum NSCS20N07D ${ }^{\top}$. Chr. I and Chr. II represent the larger and smaller chromosomes, respectively. The genes are grouped together according to the metabolic pathway. 


\section{REFERENCES}

Aziz, R. K., Bartels, D., Best, A. A., DeJongh, M., Disz, T., Edwards, R. A., et al. (2008). The RAST Server: rapid annotations using subsystems technology. BMC Genomics 9:75. doi: 10.1186/1471-2164-9-75

Baek, J. H., and Chattoraj, D. K. (2014). Chromosome I controls chromosome II replication in Vibrio cholerae. PLOS Genet. 10:e1004184. doi: 10.1371/journal. pgen.1004184

Caporaso, J. G., Kuczynski, J., Stombaugh, J., Bittinger, K., Bushman, F. D., Costello, E. K., et al. (2010). QIIME allows analysis of high-throughput community sequencing data. Nat. Methods 7, 335-336. doi: 10.1038/nmeth. f.303

Chin, C. S., Alexander, D. H., Marks, P., Klammer, A. A., Drake, J., Heiner, C., et al. (2013). Nonhybrid, finished microbial genome assemblies from long-read SMRT sequencing data. Nat. Methods 10, 563-569. doi: 10.1038/nmeth.2474

Delcher, A. L., Bratke, K. A., Powers, E. C., and Salzberg, S. L. (2007). Identifying bacterial genes and endosymbiont DNA with Glimmer. Bioinformatics 23, 673-679. doi: 10.1093/bioinformatics/btm009

DeSantis, T. Z., Hugenholtz, P., Larsen, N., Rojas, M., Brodie, E. L., Keller, K., et al. (2006). Greengenes, a chimera-checked 16S rRNA gene database and workbench compatible with ARB. Appl. Environ. Microbiol. 72, 5069-5072. doi: 10.1128/aem.03006-05

Dikow, R. B., and Smith, W. L. (2013). Genome-level homology and phylogeny of Vibrionaceae (Gammaproteobacteria: Vibrionales) with three new complete genome sequences. BMC Microbiol. 13:80. doi: 10.1186/1471-2180-13-80

Dryselius, R., Kurokawa, K., and Iida, T. (2007). Vibrionaceae, a versatile bacterial family with evolutionarily conserved variability. Res. Microbiol. 158, 479-486. doi: 10.1016/j.resmic.2007.04.007

Eid, J., Fehr, A., Gray, J., Luong, K., Lyle, J., Otto, G., et al. (2009). Real-time DNA sequencing from single polymerase molecules. Science 323, 133-138. doi: 10.1126/science.1162986

Galli, E., Poidevin, M., Le Bars, R., Desfontaines, J. M., Muresan, L., Paly, E., et al. (2016). Cell division licensing in the multi-chromosomal Vibrio cholerae bacterium. Nat. Microbiol. 1:16094. doi: 10.1038/nmicrobiol.2016.94

Gerding, M. A., Chao, M. C., Davis, B. M., and Waldor, M. K. (2015). Molecular dissection of the essential features of the origin of replication of the second Vibrio cholerae chromosome. mBio 6:e0073-15. doi: 10.1128/mBio. 00973-15

Heidelberg, J. F., Eisen, J. A., Nelson, W. C., Clayton, R. A., Gwinn, M. L., Dodson, R. J., et al. (2000). DNA sequence of both chromosomes of the cholera pathogen Vibrio cholerae. Nature 406, 477-483. doi: 10.1038/35020000

Huang, Z., Dong, C., and Shao, Z. (2016). Paraphotobacterium marinum gen. nov., sp. nov., a member of the family Vibrionaceae, isolated from surface seawater. Int. J. Syst. Evol. Microbiol. 66, 3050-3056. doi: 10.1099/ijsem.0.001142

Huang, Z., Li, X., Wang, L., and Shao, Z. (2014). Changes in the intestinal bacterial community during the growth of white shrimp, Litopenaeus vannamei. Aquacult. Res. 47, 1737-1746. doi: 10.1111/are.12628

Kanehisa, M., Sato, Y., Kawashima, M., Furumichi, M., and Tanabe, M. (2016). KEGG as a reference resource for gene and protein annotation. Nucleic Acids Res. 44, D457-D462. doi: 10.1093/nar/gkv1070
Kozich, J. J., Westcott, S. L., Baxter, N. T., Highlander, S. K., and Schloss, P. D. (2013). Development of a dual-index sequencing strategy and curation pipeline for analyzing amplicon sequence data on the MiSeq Illumina sequencing platform. Appl. Environ. Microbiol. 79, 5112-5120. doi: 10.1128/AEM.01043-13

Krzywinski, M., Schein, J., Birol, I., Connors, J., Gascoyne, R., Horsman, D., et al. (2009). Circos: an information aesthetic for comparative genomics. Genome Res. 19, 1639-1645. doi: 10.1101/gr.092759.109

Lowe, T. M., and Eddy, S. R. (1997). tRNAscan-SE: a program for improved detection of transfer RNA genes in genomic sequence. Nucleic Acids Res. 25, 955-964. doi: 10.1093/nar/25.5.0955

Okada, K., Iida, T., Kita-Tsukamoto, K., and Honda, T. (2005). Vibrios commonly possess two chromosomes. J. Bacteriol. 187, 752-757. doi: 10.1128/JB.187.2. 752-757.2005

Schloss, P. D., Westcott, S. L., Ryabin, T., Hall, J. R., Hartmann, M., Hollister, E. B., et al. (2009). Introducing mothur: open-source, platform-independent, community-supported software for describing and comparing microbial communities. Appl. Environ. Microbiol. 75, 7537-7541. doi: 10.1128/AEM. 01541-09

Skopina, M. Y., Vasileva, A. A., Pershina, E. V., and Pinevich, A. V. (2016). Diversity at low abundance: the phenomenon of the rare bacterial biosphere. Microbiology 85, 272-282. doi: 10.1134/s0026261716030139

Tagomori, K., Iida, T., and Honda, T. (2002). Comparison of genome structures of vibrios, bacteria possessing two chromosomes. J. Bacteriol. 184, 4351-4358. doi: 10.1128/JB.184.16.4351-4358.2002

Tatusov, R. L., Natale, D. A., Garkavtsev, I. V., Tatusova, T. A., Shankavaram, U. T., Rao, B. S., et al. (2001). The COG database: new developments in phylogenetic classification of proteins from complete genomes. Nucleic Acids Res. 29, 22-28. doi: 10.1093/nar/29.1.22

Teng, J. L. L., Yeung, M. L., Chan, E., Jia, L., Lin, C. H., Huang, Y., et al. (2017). PacBio but not illumina technology can achieve fast, accurate and complete closure of the high GC, complex Burkholderia pseudomallei two-chromosome genome. Front. Microbiol. 8:1448. doi: 10.3389/fmicb.2017.01448

Val, M. E., Marbouty, M., de Lemos Martins, F., Kennedy, S. P., Kemble, H., Bland, M. J., et al. (2016). A checkpoint control orchestrates the replication of the two chromosomes of Vibrio cholerae. Sci. Adv. 2:e1501914. doi: 10.1126/sciadv. 1501914

Vallenet, D., Engelen, S., Mornico, D., Cruveiller, S., Fleury, L., Lajus, A., et al. (2009). MicroScope: a platform for microbial genome annotation and comparative genomics. Database 2009:ba021. doi: 10.1093/database/bap021

Conflict of Interest Statement: The authors declare that the research was conducted in the absence of any commercial or financial relationships that could be construed as a potential conflict of interest.

Copyright (C) 2017 Huang, Liu and Shao. This is an open-access article distributed under the terms of the Creative Commons Attribution License (CC BY). The use, distribution or reproduction in other forums is permitted, provided the original author(s) or licensor are credited and that the original publication in this journal is cited, in accordance with accepted academic practice. No use, distribution or reproduction is permitted which does not comply with these terms. 